\title{
Diversity in Genetic Counseling: Strategies from the LEND Network
}

\author{
Ashley Hatchel • Janet Willis • Catherine Reiser • \\ Robert Marion - Crystal Pariseau • \\ Lauren Vanner-Nicely • Joann Bodurtha
}

Received: 20 October 2008 / Accepted: 12 November 2008 / Published online: 19 February 2009

(C) National Society of Genetic Counselors, Inc. 2008

To the Editor:

We appreciate the article published 17 June 2008, "Diversity in Genetic Counseling: Past, Present and Future," and its illumination of the issue concerning a lack of ethnic and racial diversity in genetic counseling. The lessons learned regarding the experiences of other health professions offer a solid basis for developing similar initiatives in the field of genetic counseling.

Experiences within the network of Leadership Education in Neurodevelopmental Disabilities (LEND) Programs offer additional strategies to address diversity. LEND programs are federally funded, advanced level interdisciplinary training programs committed to training leaders in the field of neurodevelopmental and related disabilities. There are currently 38 programs in the U.S.

In 2005, the Association of University Centers on Disabilities (AUCD) announced a grant opportunity within

\author{
A. Hatchel $(\bowtie) \cdot J$. Willis $\cdot$ L. Vanner-Nicely \\ Va-LEND Program, Virginia Commonwealth University, \\ Richmond, VA, USA \\ e-mail: hatchelae@vcu.edu \\ C. Reiser \\ WI-LEND Program, University of Wisconsin-Madison, \\ Madison, WI, USA

\section{R. Marion} \\ NY-LEND Program, Albert Einstein College of Medicine, \\ Bronx, NY, USA \\ C. Pariseau \\ Association of University Centers on Disabilities, \\ Silver Spring, MD, USA

\section{J. Bodurtha} \\ Human and Molecular Genetics and Va-LEND Program, \\ Virginia Commonwealth University, \\ Richmond, VA, USA
}

the LEND network to increase leadership training in genetic counseling programs, to increase the genetic content in LEND curricula and to develop innovative means to increase the number and diversity of genetic counselors serving children with special health care needs and their families. Three 3-year projects were funded. They included the LEND programs at Albert Einstein College of Medicine, University of Wisconsin, and Virginia Commonwealth University. Funding was provided through the Health Resources and Services Administration's Maternal and Child Health Bureau.

\section{The Virginia Experience}

The Virginia LEND program implemented a grant entitled, SYNERGY: Genetic Counseling and Va-LEND Working Together (SYNERGY). The purpose of SYNERGY was two-fold: 1) to improve the integration of the genetic component of health and well-being into leadership training programs nationally, and 2) to prepare a diverse workforce of genetic counselors capable of teaming with other health care professionals to provide leadership in serving children with special health care needs and disabilities. The project supported genetic counselor training in leadership and disabilities, enhanced multicultural recruitment to LEND training, disseminated learning modules in genetic awareness, family history, and newborn screening, and provided a focus for enhancing the genetic component of LEND curricula nationally.

To address the goal of increasing diversity, Va-LEND established a Multicultural Advisory Committee composed of former and current trainees of multi-racial and ethnic backgrounds, our consultants in the field of Genetic Counseling, faculty from two Historically Black Colleges 
and Universities (HBCUs) and other representatives from the University. We drew upon the resources of our community to form partnerships with two HBCUs in Virginia, specifically Virginia State University and Virginia Union University, to sponsor two undergraduate students for a summer internship each summer.

Through the grant and support of the Multicultural Advisory Committee, we have exposed six college students from HBCUs to the field of genetic counseling and disabilities. Their 8 week internship included rotations in LEND, autism and genetics clinics, weekly meetings with LEND faculty and graduate genetic counseling students, attending Pediatric Grand Rounds, meetings with a young adult with disabilities and families who have children with disabilities, preparing paper presentations on a topic related to genetics and serving as genetic counseling ambassadors for their respective HBCU. It is our hope that this internship will encourage the students to consider a career in genetic counseling or other related health care fields.

\section{The Wisconsin Experience}

The MCHLEND Genetics Project at the University of Wisconsin-Madison had similar goals to the SYNERGY project; 1) to increase the number of genetic counselors who are prepared to demonstrate leadership in serving individuals with neurodevelopmental and other developmental disabilities, 2) to increase the awareness, knowledge and skills regarding genetics and genetic counseling among LEND trainees from non-genetic disciplines and 3) to develop and implement strategies for recruiting genetic counseling students from diverse ethnic, racial and cultural backgrounds. Over the 3 year grant period all UW-Madison genetic counseling students (14) completed the LEND curriculum, and all LEND trainees from non-genetics disciplines ( 45) received genetics instruction that was based on the National Coalition for Health Professional Education in Genetics suggested core competencies for health professionals.

Wisconsin's LEND Genetics Project diversity recruitment activities included several strategies suggested by Mittman and Downs. As a state (WI) with a relatively homogeneous population it was important that Project staff connect with already established institutional resources (e.g. diversity recruitment and retention offices of both the graduate school and the medical school), and local school districts with large diverse student populations (Madison and Milwaukee Public Schools). Although response to Project staff overtures were variable, through the connections that were established specific objectives were met, most importantly: genetic counseling recruitment materials were developed, and distributed, at national conferences sponsored by various underrepresented minority student groups (e.g. Society for the Advancement of Chicano and Native American Students, American Indian Science and Engineering Society, Annual Biomedical Research Conferences for Minority Students, McNair Scholars), presentations were given at local high schools about genetic counseling as a profession and the LEND trainees and greater campus had the opportunity to hear national experts discuss cultural competence in healthcare (e.g., Tawara Goode, director of the Georgetown National Center for Cultural Competence in Healthcare). While the latter is not a specific recruitment effort, awareness of the need for cultural sensitivity in healthcare relates to a broader climate issue that Project staff also felt was important to address. Since the initiation of the Wisconsin LEND Genetics Project a student who self-identifies as from an underrepresented minority (URM) has enrolled in the Genetic Counseling Program. In addition, the newly developed campus connections led to funding this student's entire first year of training (tuition, stipend and insurance). Additionally, these same connections led to the current mentoring by the Genetic Counseling Program director of an undergraduate student from a URM group.

\section{The New York Experience}

The LEND program at Albert Einstein College of Medicine in New York noted that the efforts of their genetics grant have yielded huge success in the role of educating genetic counselors about individuals with neurodevelopmental disabilities and conversely educating LEND trainees about genetic counseling. Albert Einstein's LEND program has decided to extend its efforts beyond the funding period and is hopeful their efforts, along with those of Virginia and Wisconsin, will serve to increase both interest and diversity in the field of genetic counseling.

These are examples from the genetic counseling grants sponsored by the Association of University Centers on Disabilities in 2005-2008. The national LEND network offers their experience and expertise as possible strategies are being considered by others interested in increasing diversity in genetic counseling. Learn more about LEND and AUCD online at http://www.aucd.org. 